\title{
Erratum to: Genetic diversity of giant clams (Tridacna spp.) and their associated Symbiodinium in the central Red Sea
}

\author{
Melissa K. Pappas ${ }^{1} \cdot$ Song He $^{1} \cdot$ Royale S. Hardenstine ${ }^{1} \cdot$ Hana Kanee $^{1}$. \\ Michael L. Berumen ${ }^{1}$
}

Published online: 7 July 2017

(C) Senckenberg Gesellschaft für Naturforschung and Springer-Verlag GmbH Germany 2017

Erratum to: Mar Biodiv

DOI 10.1007/s12526-017-0715-2

Originally published article contains error. Figures 4, 5 and 6 of the original article were published with incorrect images and captions. The correct figures are shown below.

The original article has been corrected.

The online version of the original article can be found at http://dx.doi.org/ $10.1007 / \mathrm{s} 12526-017-0715-2$

Melissa K. Pappas

melissa.pappas@kaust.edu.sa

1 Red Sea Research Center, Division of Biological and Environmental Science and Engineering, King Abdullah University of Science and Technology, Thuwal 23955-6900, Saudi Arabia 

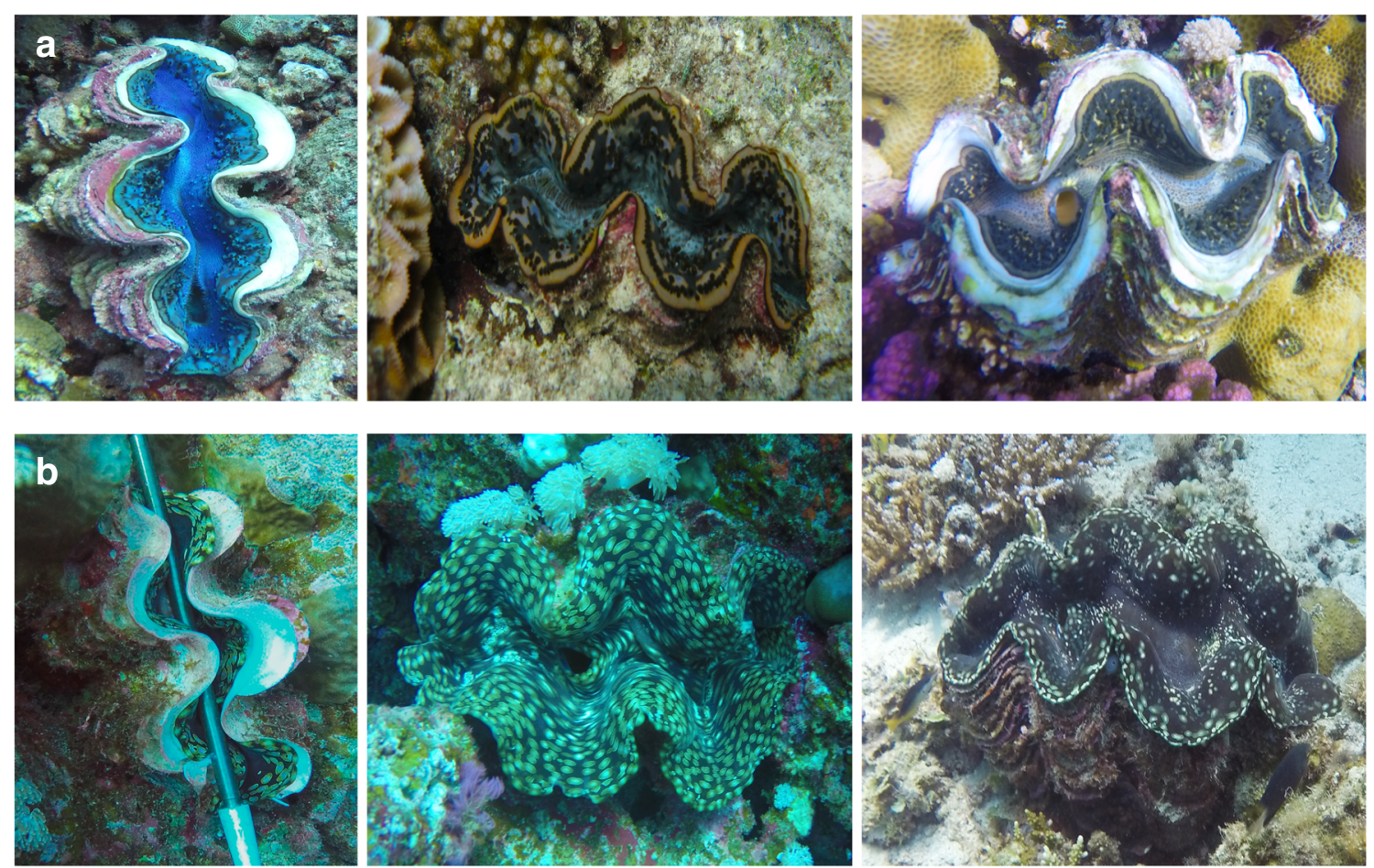

Fig. 4 Photographs taken in situ depicting the morphology and color patterns of T. maxima (row A) and T. squamosa (row B) 


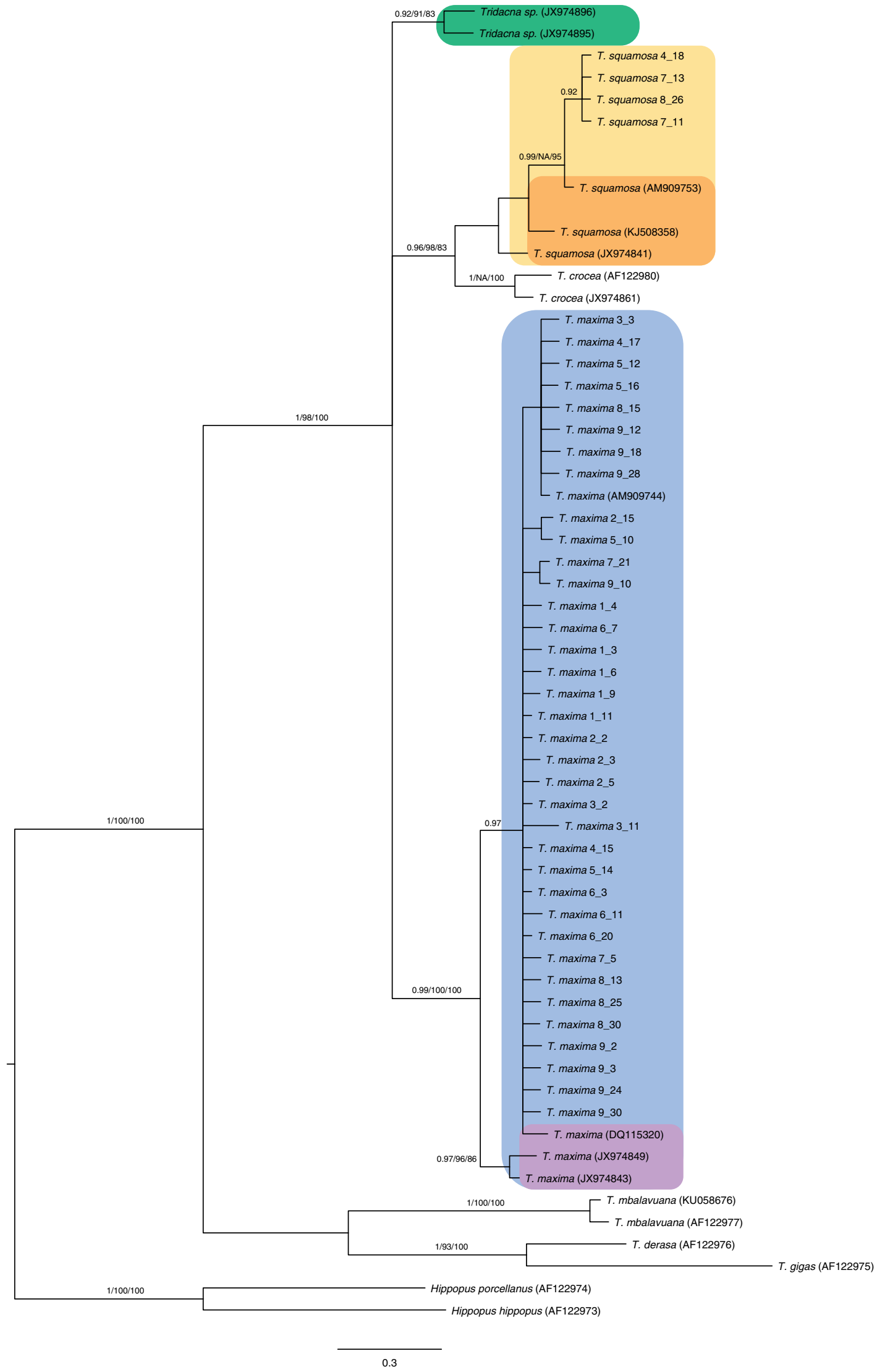

Fig. 5 Phylogenetic tree of $16 \mathrm{~S}$ tridacnid sequences from this study and references from GenBank. Branch support values are Bayesian posterior probabilities/neighbor joining bootstrap values/maximum likelihood bootstrap values with the Bayesian inference tree topology 


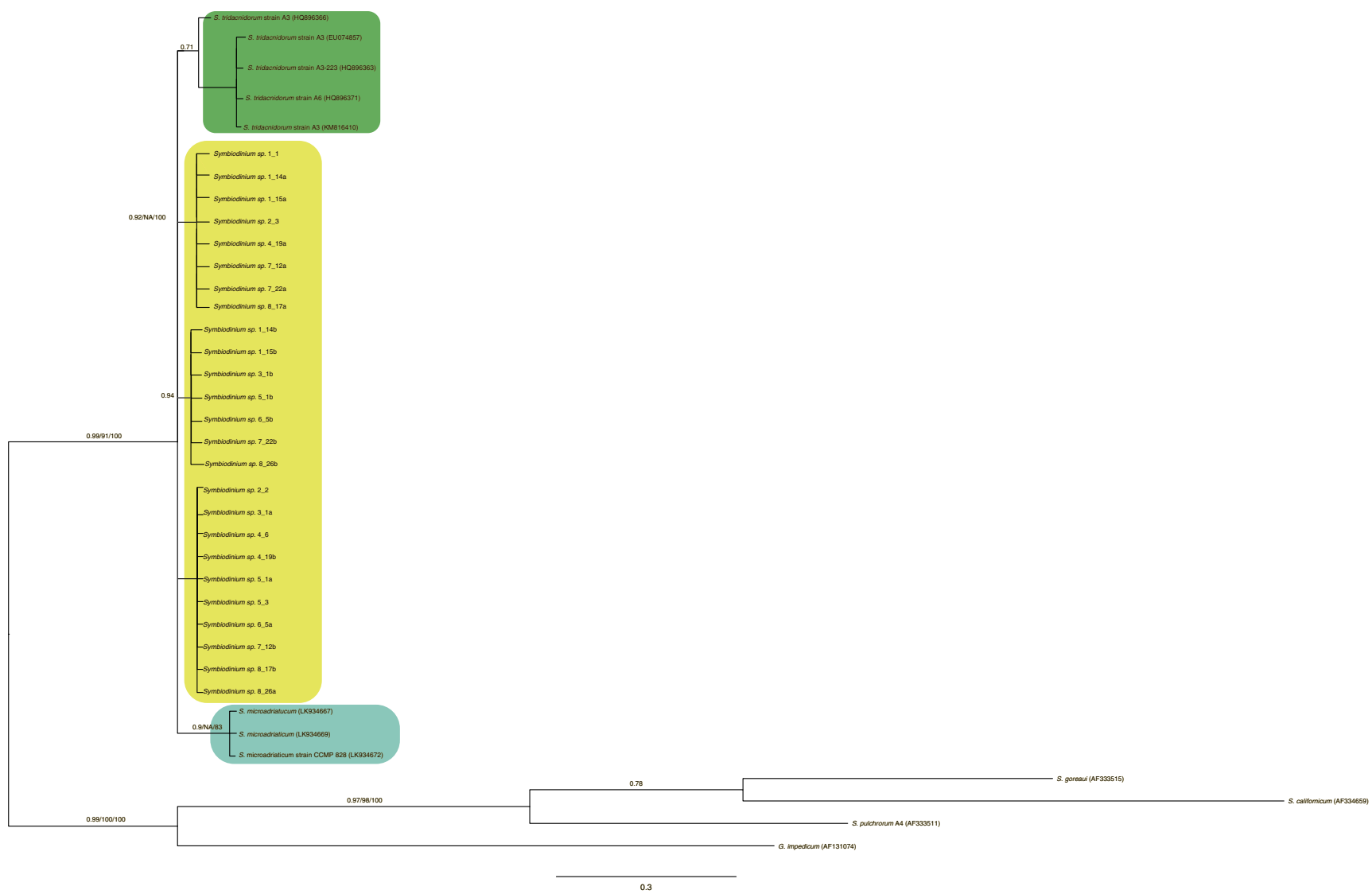

Fig. 6 Phylogenetic tree of ITS Symbiodinium sequences from this study and references from GenBank. Branch support values are Bayesian posterior probabilities/neighbor joining bootstrap values/maximum likelihood bootstrap values with the Bayesian inference tree topology 